\title{
Cryoneurolysis for the treatment of lumbar zygoapophyseal joint pain
}

\author{
Manuel Cunha e Sa $\cdot$ Sanjay Behari
}

Received: 2 February 2011 / Accepted: 3 February 2011 / Published online: 24 February 2011

(C) Springer-Verlag 2011

The myriad of treatment options available for low-back pain aptly focus on several issues:

1. The universality of the problem afflicting populations across various ages, occupations and countries.

2. The enormous burden it imposes both in terms of patient suffering and medical costs.

3. The multitude of existing mechanisms that may be responsible that often require differing treatment strategies.

4. All potential pain sources may not manifest individually but in a complex and often tandem fashion, which makes their exact identification even more elusive.

5. Pain perception itself is a complex domain greatly influenced by one's personality and various psychological factors, such as anxiety and depression.

6. None of the strategies works to take care of the lowerback pain in its entirety.

Zygaphophyseal or facet joint pain is an important and yet often missed entity in the spectrum of lower-back pain. The condition is likely to be missed due to many factors. With modern multiplaner imaging techniques like contrast enhanced magnetic resonance imaging and computed tomographic scanning, there is an increasing preoccupation to diagnose a prolapsed or degenerated intervertebral disc or osteophytosis. There is, unfortunately, no unequivocal

\footnotetext{
M. Cunha e Sa $(\bowtie)$

Department of Neurosurgery, Hospital Garcia de Orta,

Almada 2800, Portugal

e-mail: mcunhaesa@gmail.com

S. Behari

Department of Neurosurgery,

Sanjay Gandhi Postgraduate Institute,

Lucknow, UP, India
}

test to diagnose facet joint pathology, even when special emphasis is placed on concentrating on its focused and enlarged images in various sequences. The manifestation of facet joint pain closely resembles that of lumbar radiculopathy related to disc degeneration and prolapse. Unless facet joint arthropathy is suspected and tenderness of the lumbar facet joint specifically sought for on clinical examination, the entity is often missed. This is more likely in the subgroups of middle-aged women with multiple joint pains and associated facet arthropathy, and in middle-aged and elderly patients with borderline thecal compression and foraminal stenosis with degenerative disc disease or prolapse.

Knoeler and co-workers have done a yeoman service in focusing attention on the zygapophyseal joint as a specific generator of lower-back pain and the stellar role minimally invasive techniques play in effectively treating it. In a retrospective series, the authors have reported their experience in 91 of their patients having undergone 117 cryoneurolysis procedures for the treatment of pain attributable to the facet joint. This they did by utilizing the technique of thermal denervation of the facet to obtain pain relief. The level of evidence for the efficacy of denervation procedures for lumbar medial branch nerves either by radiofrequency or cryoneurolysis has been found to be moderate for short and long-term pain relief [1]. Perhaps, as opposed to the commoner techniques of chemical rhizotomy and radiofrequency thermocoagulation, where there is an inherent danger of the spread of chemical or heat respectively to the functional nerve roots in close proximity, cryotherapy has the advantage of being a safer alternative.

The scientific temper of the study is commendable and may serve as a baseline study for further research in this area. All evidence of nerve root impingement was ruled out systematically in the imaging studies obtained. Prior to 
proceeding with the actual facet joint denervation, there was a diligent establishment of the source of pain by ensuring its temporary relief with two diagnostic blocks. Psychological profiling of patients was undertaken and those who were depressed were identified. The patient population where facet joint cryotherapy constituted a seminal procedure was compared with that where the procedure was performed after other interventions had already failed. Thus, those patients in whom this procedure was not likely to meet the expected goals could be identified.

The enthusiasm for adopting the results of the study in clinical practice, however, should be tempered, bearing in mind its inevitable shortcomings. No appreciation of the morphologic changes affecting the facet joints and the remaining components of the spinal unit is included. There is no mention of any functional studies that are possible and all imaging was probably obtained in the recumbent position. Without a control group, a recurring thought that incessantly prevails is that the placebo effect of the procedure may have been, to some extent, responsible for the good results achieved. In spite of the limited benefit extracted from facet joint cryotherapy in patients who were psychologically affected and those with persisting lowerback pain despite the recurrent use of both percutaneous and open techniques, the authors still persisted in recommending the use of this procedure in these subgroups. An inevitable conclusion derived from the fact that there was no statistically significant difference in the pre- and post- interventional pain rating between patients with and without prior spine surgery, with spine surgery at any level and with spine surgery at the same level may be that in failed back syndrome following surgery this procedure may not have a defining role. The most significant drawback of this study, however, is the high drop-out rate of patients who actually responded to the follow-up questionnaire from amongst the total number of patients undergoing facet joint cryotherapy. An implication that may significantly impact upon outcome may be that patients who did not benefit from therapy or were worse off with it did not respond to the questionnaire.

Nevertheless, this study highlights the efficacy and lesser invasiveness of the percutaneous pain procedure of facet joint cryoneurolysis compared with other surgical solutions (minimally invasive or not). It is evident that the former procedure ought to be included as an integral part of the initial levels of the step-ladder approach while considering the armamentarium of procedures existing for the treatment of lower-back pain.

Conflicts of interest M. Cunha e Sá is consultant for Medtronic.

\section{References}

1. Boswell M, Colson J, Sehgal N, Dunbar E, Epter R (2007) A systematic review of therapeutic facet joint interventions in chronic spinal pain. Pain Physician 10:229-253 